\section{Quantitative Magnetic Resonance Imaging Has Potential for Assessment of Spondyloarthritis: Arguments for its Study and Use}

\section{To the Editor:}

We read with interest the recent paper on diffusion-weighted imaging (DWI) as a means of supporting the diagnosis of ankylosing spondylitis ${ }^{1}$, and the corresponding editorial questioning the role of DWI ${ }^{2}$. The paper ${ }^{1}$ adds to the growing body of evidence supporting the use of DWI to characterize and quantify inflammation in patients with spondyloarthritis $(\mathrm{SpA})^{3,4}$. The corresponding editorial argues that DWI is of limited clinical use, and the authors' main criticisms are summarized as follows:

1. Any sequence can be measured numerically, and this is a facility not confined to DWI.

2. The number of steps required for normalizing measurements and choosing regions of interest (ROI) depends on review of the initial qualitative images and is subjective.

3. The variable diffusion of background marrow poses further challenges for DWI.

However, if we look in greater detail at the existing literature on quantitative imaging, it is apparent that many of these issues can be addressed, and indeed there are multiple advantages that quantitative techniques offer over standard sequences. Considering the criticisms in the editorial in turn:

1. In a conventional magnetic resonance image (MRI), the signal intensities are influenced by the composition of tissue but also by hardware-related factors such as the spatial variations in the sensitivity of the receiving coil. This means that the biological information contained in the image is "confounded" by these hardware factors and is not directly comparable between scanners or between repeat scans ${ }^{5}$. The confounding effect of acquisition variables is, in general, true of all conventional MR imaging. However, quantitative MRI methods typically acquire a series of images, allowing us to fit a model to the acquired data and to calculate an objective measure [e.g., apparent diffusion coefficient (ADC)] that reflects intrinsic tissue properties, and is largely independent of these confounding factors ${ }^{5}$.

Quantitative imaging is now a huge field ${ }^{5,6,7}$, and much of the research performed within this field is founded on the greater objectivity of quantitative MRI methods. There are multiple organizations dedicated to the oversight of quantitative imaging biomarker (QIB) development ${ }^{7,8}$. A key component of the definition of a QIB is that it should be objectively measured $^{7}$; it is misleading to say that, by drawing ROI, a conventional MR image can be quantified in the same way.

2. The number of steps adopted by the authors of the paper appears to us to be performed to allow direct comparison of DWI measurement with Spondyloarthritis Research Consortium of Canada scoring. The methodology for determining ROI is somewhat cumbersome and its criticism in the context of potential clinical use is justified. However, it is often necessary to start with relatively basic techniques during the development of new methods. Further, there are other potential approaches to interpreting quantitative images in a clinically relevant way. ROI do not need be focal; an alternative approach could be to sample the whole of the sacroiliac joint, and then analyze this data using thresholding and histographic analysis ${ }^{9}$. This is less subjective that manually defining ROI. In the future, more sophisticated segmentation techniques may enable automatic separation of inflamed areas from normal marrow, removing the subjective element altogether ${ }^{10}$.

3. The observation in the published paper that background marrow in patients in the pretreatment group had higher ADC values than patients with nonradiographic AS or chronic back pain is of interest ${ }^{1}$. Rather than being a challenge, this is a question. Why is this so, and is this a reproducible finding in larger numbers of patients? One advantage of quantitative methods is that they allow us to ask more direct questions of the underlying physiology. Further, there are already methods in other fields (e.g., lesion segmentation in multiple sclerosis) that allow for variable thresholds in the quantification of pathology, which seem well-suited to address this problem.

A particular advantage of quantitative MRI is that multiple techniques, each reflecting different but known aspects of tissue physiology, such as fat fraction, perfusion and diffusion, can be combined as "multiparametric" imaging. This enables us to examine different aspects of tissue physiology, the "virtual imaging biopsy." This in turn may improve our understanding of pathophysiology and enable us to examine the biology underpinning therapeutic response in a way that conventional qualitative imaging cannot.

Quantitative imaging is in its infancy. There are many problems remaining including acquiring consistent and comparable data across platforms and sites, defining pathological values, and validating these in different disease states. Criticism of its current relevance to clinical imaging of bone marrow is legitimate, and it is still necessary to demonstrate additional benefit when combined with conventional imaging. However, in the longer term, it seems plausible that quantitative MRI will one day be a powerful tool for the assessment of disease activity in inflammatory conditions. Clearly, further study of this area is needed.

MARGARET ANNE HALL-CRAGGS (-), MD, Professor of Medical Imaging, Centre for Medical Imaging, Division of Medicine, University College London (UCL) and Consultant Radiologist, UCL Hospitals (UCLH); TIMOTHY JAMES PENGILLY BRAY — , PhD, Hon. Clinical Lecturer, Centre for Medical Imaging, Division of Medicine, UCL and Specialist Registrar, UCLH; COZIANA CIURTIN — , PhD, Associate Professor, Arthritis Research UK Centre for Adolescent Rheumatology, UCL; ALAN BAINBRIDGE 10 , PhD, Head of Medical Physics, UCLH, and Centre for Medical Imaging, Division of Medicine, UCL. MHC and CC are supported by the UCLH UK National Institute for Health Research UCLH Biomedical Research Centre. TJB is supported by Arthritis Research UK, grant number 21369. Address correspondence to Prof. M.A. Hall-Craggs, 2nd Floor, Charles Bell House, 43-45 Foley St., London W1W 7TS, UK. E-mail: margaret.hall-craggs@nhs.net

\section{REFERENCES}

1. Bradbury LA, Hollis KA, Gautier B, Shankaranarayana S, Robinson PC, Saad N, et al. Diffusion-weighted imaging is a sensitive and specific magnetic resonance sequence in the diagnosis of ankylosing spondylitis. J Rheumatol 2018;45:771-8.

2. Lambert RG, Maksymowych WP. Diffusion-weighted imaging in axial spondyloarthritis: a measure of effusion or does it elicit confusion? J Rheumatol 2018;45:729-30.

3. Gezmis E, Donmez FY, Agildere M. Diagnosis of early sacroiliitis in seronegative spondyloarthropathies by DWI and correlation of clinical and laboratory findings with ADC values. Eur J Radiol 2013;82:2316-21.

4. J P Bray T, Vendhan K, Ambrose N, Atkinson D, Punwani S, Fisher $\mathrm{C}$, et al. Diffusion-weighted imaging is a sensitive biomarker of response to biologic therapy in enthesitis-related arthritis. Rheumatology 2017;56:399-407.

5. Tofts PS, Davies GR, Dehmeshki J. Histograms: measuring subtle diffuse disease. In: Tofts P, editor. Quantitative MRI of the brain: measuring changes caused by disease. Philadelphia: J. Wiley \& Sons; 2005:581-610.

6. O'Connor JP, Aboagye EO, Adams JE, Aerts HJ, Barrington SF, Beer AJ, et al. Imaging biomarker roadmap for cancer studies. Nat Rev Clin Oncol 2017;14:169-86.

7. Sullivan DC, Obuchowski NA, Kessler LG, Raunig DL, Gatsonis C, Kondratovich M, et al. Metrology standards for quantitative imaging. Radiology 2015;277:813-25.

8. Taouli B, Beer AJ, Chenevert T, Collins D, Lehman C, Matos C, et al. Diffusion-weighted imaging outside the brain: consensus statement from an ISMRM-sponsored workshop. J Magn Reson Imaging 2016;44:521-40.

9. Bray TJP, Dudek A, Sakai N, Rajesparan K, Fisher C, Ciurtin C, et al. The BEACH tool: a quantitative MRI-based method for measuring oedema and fat metaplasia in spondyloarthritis. Ann Rheum Dis 2018;77 Suppl:A821.

10. Blackledge MD, Collins DJ, Tunariu N, Orton MR, Padhani AR, 
Leach MO, et al. Assessment of treatment response by total tumor volume and global apparent diffusion coefficient using

diffusion-weighted MRI in patients with metastatic bone disease: A feasibility study. PLoS One 2014;9:e91779.

J Rheumatol 2019;46:5; doi:10.3899/jrheum.181049 